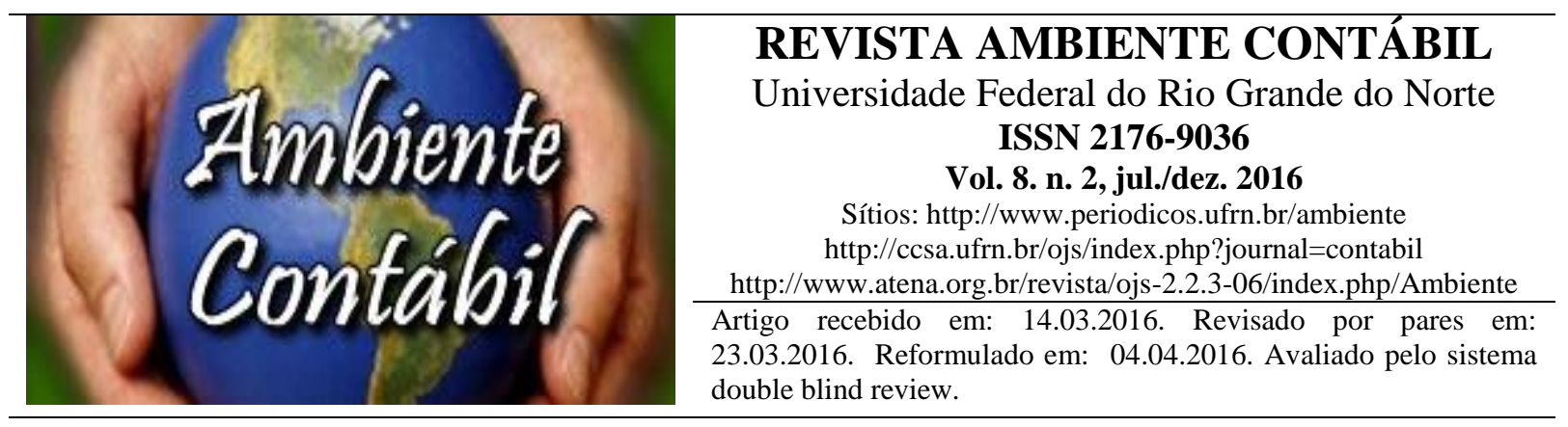

\title{
UM ESTUDO COMPARATIVO DA EFICIÊNCIA DE DISTRIBUIDORAS BRASILEIRAS DE ENERGIA ELÉTRICA PRIVADAS E PÚBLICAS
}

\section{A COMPARATIVE STUDY OF THE EFFICIENCY OF PRIVATE AND PUBLIC BRAZILIAN ELECTRICITY UTILITIES}

\section{UN ESTUDIO COMPARATIVO DE LA EFICIENCIA DE LAS DISTRIBUIDORAS BRASILEÑAS DE ENERGÍA ELÉCTRICA PRIVADAS Y PÚBLICAS}

\section{Autores}

\section{Diego Rodrigues Boente}

Doutorando em Ciências Contábeis (UnB/UFPB/UFRN). Mestre em Ciências Contábeis (UnB/UFPB/UFRN). Analista de Finanças e Controle e Gerente de Normas e Procedimentos

Contábeis (GENOC) da Coordenação-Geral de Normas de Contabilidade Aplicadas à Federação (CCONF) da Secretaria do Tesouro Nacional/MF. Endereço: Setor de Autarquias Sul - Quadra 3 - Edifício Órgãos Regionais - Asa Sul CEP: 70079-900 - Brasília, DF - Brasil - Telefone: (61) 3412-4905

E-mail: diegoboente@gmail.com

\section{Paulo Roberto Barbosa Lustosa}

Doutor em Contabilidade e Controladoria pela Universidade de São Paulo (USP). Professor do Departamento de Ciências Contábeis e Atuariais da Universidade de Brasília (UnB). Endereço: Campus Universitário Darcy Ribeiro - Asa Norte CEP: 70910-900 - Brasília, DF Brasil - Telefone: (61) 3107-0798

E-mail: prblustosa@gmail.com

\section{RESUMO}

O presente estudo teve como foco a comparação de eficiência de entidades privadas e públicas do segmento de distribuição de energia elétrica. Para tanto, foi realizado o cálculo dos scores de eficiência através do modelo DEA-CRS, orientado a input, e, em seguida, aplicado o Teste Mann-Whitney-Wilcoxon, para identificar eventuais diferenças. A presente pesquisa foi realizada com as 62 concessionárias brasileiras de distribuição de energia elétrica. A eficiência no desempenho econômico-financeiro dessas entidades depende, sobretudo, da gestão eficiente dos recursos, que refletem diretamente nos índices que determinam sua eficiência. A análise dos dados e o resultado do teste sugere uma interpretação que as entidades privadas possuem mediana dos scores de eficiência diferente as entidades públicas. Os resultados, porém, devem ser interpretados com cautela à luz das variáveis de controle como porte e região de atuação.

Palavras-chave: Eficiência. Setor elétrico. Entidades privadas e públicas. 


\begin{abstract}
This study aims a comparison of efficiency of private and public utilities. For this, we performed the calculation of scores of efficiency through DEA-CRS model-driven input, and then we applied the Mann-Whitney-Wilcoxon test to identify any differences. This survey was conducted with 62 Brazilian utilities of electricity distribution. Efficiency in the financial performance of these entities depends, above all, efficient resources management, which reflect directly in its efficiency index. Data analysis and the test result suggest an interpretation that private entities have, on average, a different efficiency and higher public entitites. However, the results should be interpreted with caution as control variables as firm size and area of operation.
\end{abstract}

Keywords: Efficiency. Electricity sector. Private and public entities.

\title{
RESUMEN
}

Este estudio se centró en la comparación de la eficiencia de las entidades privadas y públicas en el segmento de distribución de energía eléctrica. Por lo tanto, se realiza el cálculo de las puntuaciones de la eficiencia DEA-CRS dirigido a la entrada, a continuación, aplica la prueba de Mann-Whitney-Wilcoxon para identificar cualquier diferencia. Esta investigación se llevó a cabo con 62 empresas de servicios públicos brasileños de distribución de energía eléctrica. La eficiencia en el desempeño financiero de estas entidades depende, sobre todo, la gestión eficiente de los recursos, lo que refleja directamente en los índices que determinan su eficacia. Análisis de datos y el resultado de la prueba sugiere una interpretación que las entidades privadas tienen índices de eficiencia mediana diferentes de las entidades públicas. Los resultados, sin embargo, deben ser interpretados con precaución en función de las variables de control como el tamaño y área de operaciones.

Palabras clave: Eficiencia. Sector eléctrico. Entidades públicas y privadas.

\section{INTRODUÇÃO}

O setor de energia elétrica, como utilidade pública e agente indutor dos demais setores da economia, condiciona características importantes para a análise de seu desempenho econômico-financeiro. Frequentemente, associa-se o desenvolvimento da economia de um país aos níveis de oferta e de demanda de energia elétrica.

A teoria econômica clássica preceitua que a livre concorrência produz o maior nível de bem-estar possível comparado a qualquer outra forma de mercado. Mas não é o caso do setor de energia elétrica, especificamente o segmento de distribuição, que é sujeito à falha de mercado tradicionalmente conhecida como monopólio natural. Quando o máximo nível de produção exige a presença de um único produtor, o Governo deve garantir que essa empresa não utilize seu poder monopolista para gerar lucros excessivos ou para restringir quantidade e qualidade dos serviços prestados (DEMSETZ, 1968; JARREL, 1978).

Acrescente-se ainda que, nesse setor, a incerteza associada ao longo período de maturação dos projetos (construção de subestações, ampliação da rede elétrica, etc.) e a possibilidade de mudanças de condições ao longo desse período reduzem os incentivos ao investimento de capitais privados e públicos no setor (BAUMOL; WILLING, 1981).

A partir dos anos 1990, alguns estudos têm concentrado sua análise em segmentos de transmissão e distribuição. Isso se deve, em parte, a separação estrutural do setor ocorrido no período em diversos países, mas também por questões como monopólios naturais e privatizações (ABBOTT, 2005).

No Brasil, as entidades reguladas operam em diferentes regiões e em diferentes condições operacionais e ambientais. Parte-se da premissa que o modelo de gestão da firma 
possa afetar a eficiência dessas entidades. Entretanto, a heterogeneidade é tratada de forma distinta nos vários modelos utilizados pelo órgão regulador, o que pode resultar em estimativas de custos operacionais discrepantes. Sobre esse ponto, há poucos avanços no conhecimento científico, lacuna a qual este trabalho procura preencher.

Como a estimativa de custos operacionais definida pelo regulador afeta $\mathrm{o}$ estabelecimento da tarifa de energia elétrica, um erro de estimativa pode conduzir a um ônus excessivo sobre a sociedade, se os custos forem sobre-estimados, ou um ônus excessivo sobre as entidades reguladas, se os custos forem subestimados. Portanto, são necessários mais estudos que contribuam para a compreensão e a discussão das variáveis que influenciam a eficiência dos agentes, como fator de aberto interesse pelos agentes econômicos, pelos reguladores e pela sociedade.

Considerando todo o exposto anteriormente, esse estudo se orienta pela seguinte questão problema: há diferença significativa no score de eficiência entre entidades privadas e públicas?

Portanto, o presente estudo teve como foco a comparação de eficiência de entidades privadas e públicas do segmento de distribuição de energia elétrica. O estudo busca trazer contribuições de cunho teórico e metodológico através do cálculo dos scores de eficiência através do modelo DEA-CRS, orientado a input, e, em seguida, aplicado o Teste MannWhitney-Wilcoxon, para identificar eventuais diferenças. Uma eventual diferença de eficiências entre essas entidades sugere um indicativo que este fator deva ser considerado nos modelos utilizados pelo regulador quando do estabelecimento do seu modelo tarifário.

O estudo utiliza a base de dados consolidada na Agência Nacional de Energia Elétrica (Aneel), órgão regulador brasileiro, de 2010 a 2014. Em princípio, todas as entidades são obrigadas a divulgar informações que representem adequadamente a situação econômicofinanceira das concessionárias de distribuição de energia elétrica por força da Resolução Aneel n ${ }^{\circ}$ 396/2010. Contudo, dados anteriores a esse período não foram disponibilizados pelo órgão regulador.

Além dessa introdução, o estudo foi estruturado em quatro outras seções. Na seção 2, são apresentados uma breve contextualização do modelo de regulação do setor elétrico brasileiro e estudos sobre a eficiência de entidades privadas e públicas. Os procedimentos metodológicos adotados no estudo são apresentados na seção 3. Na seção 4, são apresentadas a análise e a interpretação dos resultados. As conclusões são apresentadas na seção 5, seguidas das referências utilizadas.

\section{REFERENCIAL TEÓRICO}

\subsection{BREVE CONTEXTUALIZAÇÃO DO MODELO DE REGULAÇÃO DO SETOR ELÉTRICO BRASILEIRO}

No Brasil, o processo de reforma do setor elétrico baseou-se na mudança de paradigma da própria atividade. Até meados dos anos 1990, o paradigma envolvia a integração vertical dos diversos segmentos (geração, transmissão, distribuição e comercialização), todos conduzidos pelo Poder Público.

Com a Lei n. 9.427, de 26 de dezembro de 1996, foi criada a Agência Nacional de Energia Elétrica - Aneel, uma autarquia em regime especial, vinculada ao Ministério de Minas e Energia (MME). A Aneel tem a missão de proporcionar condições favoráveis para que o mercado de energia elétrica se desenvolva com equilíbrio entre os agentes e em benefício da sociedade (Aneel, 2015).

A partir do ano de 2004, o então modelo institucional definiu a criação de uma entidade responsável pelo planejamento do setor elétrico a longo prazo, a Empresa de Pesquisa Energética (EPE); uma instituição com a função de avaliar permanentemente a 
segurança do suprimento de energia elétrica, o Comitê de Monitoramento do Setor Elétrico (CMSE); e uma instituição para dar continuidade às atividades do Mercado Atacadista de Energia (MAE), relativas à comercialização de energia elétrica no Sistema Interligado Nacional (SIN), e a Câmara de Comercialização de Energia Elétrica (CCEE).

Também se definiu o exercício do poder concedente ao Ministério de Minas e Energia (MME) e a ampliação da autonomia do Operador Nacional do Sistema Elétrico (ONS).

Outra importante alteração foi introduzida com a Lei n. 10.848, de 15 de março de 2004, exigindo que as concessionárias, permissionárias e autorizadas de serviço público de distribuição de energia elétrica, atuando no Sistema Integrado Nacional (SIN), não possam desenvolver atividades de: geração de energia elétrica; transmissão de energia elétrica; venda de energia a consumidores, salvo algumas exceções; participação em outras sociedades de forma direta ou indireta.

Na prática, isso implicou a desverticalização societária de diversas entidades, fazendo com que as informações econômico-financeiras, antes agregadas pela combinação de operações (geração, transmissão, distribuição e comercialização), passassem a refletir cada uma separadamente.

O objetivo original que conduziu o processo de reforma foi maximizar os benefícios para a sociedade e o estabelecimento de condições econômicas eficientes no setor, criando mercados competitivos em todos os segmentos possíveis e regulações de eficiência onde não fosse possível. O propósito tem alcançado o desenvolvimento de uma energia de distribuição de baixo custo, com qualidade de serviço, aumentando o uso eficiente e reduzindo perdas.

Os governos têm investido fortemente na iniciativa privada, envolvendo funções tradicionais de investimento e funções regulatórias. Isto permitiu que companhias, resultantes de cisão de estatais, fornecessem energia elétrica para nichos de consumidores (industriais, por exemplo). Por outro lado, surgiram algumas companhias que se integraram a grupos de multinacionais estrangeiras, principalmente europeias (RUDNICK; ZOLEZZI, 2001).

Em relação ao segmento de distribuição no Brasil, o processo de reforma introduziu princípios de um "pseudo" mercado competitivo. O legislador considerou que era socialmente conveniente para esta atividade desenvolver monopólios geográficos, dado a uma relevante economia de escala da tecnologia de distribuição, o que possibilita que o custo individual de uma empresa diminui com o aumento da densidade população na área de atuação.

O maior desafio da regulação na atividade de distribuição é garantir que a escolha da tarifa permita a companhia cobrir seus custos mais uma taxa de retorno razoável ao mesmo tempo em que promova incentivos para atingir maior eficiência. Esquemas regulatórios têm sido desenvolvidos para estimular eficiências entre os agentes, sob o que tem sido chamado de regulação por benchmarking.

Basicamente, é produzir uma competição artificial entre os agentes monopolistas e uma referência de eficiência, um modelo do tipo yardstick competition, proposto por Shleifer (1985). Isto corresponde a uma competição por comparação, onde a taxa de retorno de cada distribuidor não está assegurada nem limitada, mas depende dos seus indicadores de eficiência comparada com um modelo de referência, base para o cálculo da tarifa.

As principais críticas desta metodologia envolvem a complexidade da aplicação e o argumento que os ganhos de eficiência obtidos nem sempre são compartilhados com os consumidores finais.

\subsection{ESTUDOS SOBRE A ANÁLISE DE EFICIÊNCIA DE ENTIDADES PRIVADAS E PÚBLICAS}

A análise de eficiência no setor elétrico torna-se fundamental para o planejamento de projetos de sustentabilidade do fornecimento de energia no longo prazo e o alcance de metas e 
melhorias para uma boa gestão. Essa análise torna-se imprescindível quando o ambiente é sujeito a falhas de mercado (monopólios naturais, por exemplo), que demanda a atuação da regulação como um mecanismo para restaurar o potencial de eficiência na operação dos mercados. Assim, alguns estudos avançaram nessa discussão, conforme visto a seguir.

Para Megginson (2005), existem diversas razões para que um Estado lance mão de privatizações, mas a mais importante para que uma empresa estatal seja transferida para o setor privado é, sem dúvida, a insatisfação da sociedade com o desempenho operacional apresentado e a perspectiva de que os investidores privados possam promover o desenvolvimento e ampliação dos serviços, introduzindo significativos ganhos na qualidade do serviço prestado e, por consequência, desonerando o Estado dessa tarefa. O risco nesses casos é que na presença de monopólios naturais, as empresas privadas possam agir segundo seus interesses privados, prejudicando ou apropriando-se de parte do bem-estar social de modo a maximizar seus resultados.

E por que se esperaria melhor desempenho das entidades enquanto privadas que enquanto públicas? Para Lima (1997), o seu desempenho eficiente é claramente um bem público (bem cujo consumo por um indivíduo, não exclui o consumo de outro indivíduo simultaneamente). $\mathrm{O}$ conjunto da sociedade será beneficiado, mas cada cidadão individualmente terá um benefício muito pequeno. Além disso custo de um monitoramento dessas entidades é muito alto para cada cidadão individualmente. Os governantes, entretanto, têm incentivos mais forte para tentar monitorar as entidades públicas de acordo com o interesse público. $\mathrm{O}$ primeiro deles é que uma performance eficiente das empresas pode ser utilizada como instrumento de marketing político do governo. Outro incentivo é que um desempenho financeiro satisfatório pode contribuir para um maior equilíbrio das contas públicas.

Para Vickers e Yarrow (1988), os administradores públicos poderão implementar políticas que maximizarão o bem-estar social, pois sem a competição privada a firma poderá ajustar melhor os preços do serviço ao verdadeiro custo social, proporcionando significativos ganhos com as externalidades produzidas.

Shapiro e Willig (1990) defendem que o modelo de gestão pública proporciona uma função de utilidade que concilia o interesse social e o bem-estar privado, esse último refletindo o interesse individual e as melhorias proporcionadas pelas políticas públicas desenvolvidas ao longo do tempo. Quando a gestão política é eficiente, os administradores das empresas públicas são forçados a maximizar o bem-estar social, bem como o interesse do negócio sob a ótica do Estado.

Contudo, Kessler (2006) ressalta que, em geral, a prática mostra que as empresas públicas estão fortemente expostas ao risco de desvio de função provocado pelo interesse político, suportando, nesses casos, despesas e investimentos que traduzem o interesse de grupos específicos e não os interesses da sociedade em geral.

A entidade privada, por outro lado, tem como principal incentivo o lucro. Hanke (1991) argumenta que a empresa é de propriedade de indivíduos que estão livres para usar e transferir, dentro dos limites da lei, seus ativos. Consequentemente, eles possuem interesse residual no valor desses ativos e, em última instância, podem assumir obrigações em caso de falência.

Conforme Kessler (2006), aumentos de receita e redução de custos retornam maiores dividendos para os acionistas. Assim, há um forte incentivo para que os administradores maximizem os resultados e invistam em aumento de eficiência e produtividade no desenvolvimento de novos e melhores produtos.

Bubicz et al (2014) ressalva que, para muitas empresas, além do problema ambiental, o custo da energia elétrica é elevado e o aumento da eficiência energética pode ser benéfico. 
No entanto, alterar a forma de contratação para fontes incentivadas requer planejamento e investimentos.

As evidências empíricas não são conclusivas sobre uma prevalência na performance de um ou outro modelo de gestão. Por exemplo, Yarrow (1986) resenha vários 28 trabalhos realizados que tentaram comparar as diferenças de performance entre empresas públicas. Deles, 17 concluíram serem as empresas privadas mais eficientes, 6, as empresas públicas e 5 deram resultado indiferente ou ambíguo.

Koh, Berg e Kenny (1995) conduziram um estudo com hidrelétricas americanas e concluíram que as entidades públicas são mais eficientes que as privadas quando o nível de geração de energia é baixo, e menos eficientes na situação inversa. Resultados semelhantes também foram encontrados no estudo de Arocena e Price (2002) nas empresas geradoras de energia da Espanha.

Sampaio, Ramos e Sampaio (2005) investigaram a transição do setor brasileiro de geração de energia elétrica do modelo estatal para o privado visa garantir recursos para construção de novas usinas hidrelétricas e concluíram que o setor público foi mais eficiente que o privado no período analisado.

Especificamente no segmento de distribuição de energia elétrica, Bagdadioglu, Price e Weyman-Jones (1996) encontraram que os melhores scores de eficiência são de distribuidoras privadas na Turquia. Contudo, ressaltaram que não necessariamente implica sucesso do modelo de gestão privado na distribuição de energia elétrica, pois também existem organizações públicas eficientes.

Os resultados são consistentes com os achados de Kumbahkar e Hjalmarsson (1996) que conduziram estudo semelhante nas distribuidoras de energia elétrica da Suécia entre os 1970 a 1990.

\section{PROCEDIMENTOS METODOLÓGICOS}

\subsection{CARACTERIZAÇÃO DA PESQUISA}

Esta seção descreve os procedimentos metodológicos adotados no estudo. Esta pesquisa caracteriza-se como empírico-analítica sendo descritiva em relação aos objetivos, com abordagem principalmente quantitativa na análise dos dados.

\subsection{COLETA E BASE DE DADOS}

Adotou-se como população todas as 63 concessionárias brasileiras de energia elétrica (para fins deste estudo, são chamadas entidades), de acordo com dados da Aneel. No entanto, por limitações de dados, uma entidade foi retirada para compor a amostra, perfazendo o total de 62 entidades. Essas entidades possuem várias formas de organização jurídica: sociedade anônima, sociedade limitada, cooperativa e autarquia (órgão público).

O período analisado é de 2010 a 2014. Em princípio, todas as entidades são obrigadas a divulgar informações que representem adequadamente a situação econômico-financeira das concessionárias de distribuição de energia elétrica por força da Resolução Aneel no 396/2010. Entre os dados divulgados, estão as Demonstrações Contábeis Regulatórias - DCRs dessas concessionárias e permissionárias. Os números são informados pelos próprios agentes. Dados anteriores a esse período não foram disponibilizados pelo órgão regulador.

Essas entidades atualizam suas informações, provendo informações detalhadas sobre aspectos operacionais e financeiros. Isto inclui, por exemplo, custos operacionais, o número de clientes atendidos, a extensão da rede, a energia demandada, entre outras. Ressalva-se que o fenômeno em estudo trata de influências sobre a eficiência em um espaço de tempo específico (2010-2014). Este fenômeno, contudo, ocorre continuamente ao longo do tempo, cujos efeitos podem ter sido ou não captados pela janela de tempo selecionada. Com uma 
janela de tempo curta, não foi possível traçar adequadamente uma tendência sobre o efeito do modelo de gestão sobre a eficiência. Estudos posteriores poderão investigar nesse sentido.

\subsection{ANÁLISE E INTERPRETAÇÃO DOS DADOS}

Para a análise dos dados também foram coletadas as informações técnicas sobre os atributos físico-elétricos dos agentes. Esses atributos são informações utilizadas para calcular os scores de eficiência. As variáveis que compõem o cálculo do indicador de eficiência são as mesmas estabelecidas no modelo da ANEEL, conforme Resolução Aneel n ${ }^{\circ}$ 457/2011, ou seja, três produtos (outputs) e um insumo (input). A Tabela 1 sumariza as variáveis selecionadas. Todas as variáveis são quantitativas e positivas. Isso permite a eliminação de zeros existentes na base de dados, o que é importante para o cálculo do indicador de eficiência.

Tabela 1 - Variáveis selecionadas para o cálculo do score de eficiência

\begin{tabular}{c|c|c|l}
\hline \multicolumn{1}{c|}{ Indicadores } & I/O & Sigla & \multicolumn{1}{c}{ Descrição } \\
\hline Custos operacionais & Input & OPEX & $\begin{array}{l}\text { Custos com pessoal, materiais, serviços e } \\
\text { outros custos operacionais. }\end{array}$ \\
\hline Número de clientes & Output & CL & Número de clientes atendidos em um ano. \\
\hline Energia distribuída & Output & ED & $\begin{array}{l}\text { Quantidade de energia em MWh demandada } \\
\text { em um ano. }\end{array}$ \\
\hline Densidade da rede & Output & DR & $\begin{array}{l}\text { Quantidade de linhas de distribuição }(\mathrm{km}) \\
\text { dividida pela área de concessão (km²). }\end{array}$ \\
\hline
\end{tabular}

Fonte: Elaborado pelos autores.

Selecionadas as variáveis, as resultantes medidas de eficiência são avaliadas com o uso dos escores fornecido pelo método de benchmarking da Análise Envoltória de Dados (DEA) para os anos 2010 a 2014.

Os métodos de benchmarking podem ser definidos como um processo comparação de alguma medida de desempenho em relação a uma referência (FARSI; FILIPPINI; GREENE, 2006). A eficiência comumente tem como medida é a distância em relação a uma fronteira de custo, formada pelo potencial de alocação dos inputs. A estimativa da eficiência pode ser realizada utilizando uma grande variedade de modelos, mas classificadas em duas categorias principais de paramétricos e não paramétricos.

A abordagem não-paramétrica, como a Análise Envoltória de Dados (DEA), usa programação linear para determinar a eficiência de fronteira de uma empresa. Nessa abordagem a fronteira custo é considerada como uma função determinística das variáveis observadas é mais fácil de estimar usando poucas observações.

DEA é um método não-paramétrico e usa programação linear para calcular a fronteira de eficiência ou melhores práticas de uma amostra (FARRELL, 1957). As unidades de tomada de decisão (DMU) eficientes formam a fronteira "envelope" que contém as entidades ineficientes. A eficiência das empresas é calculada em termos de pontuação em uma escala de 0 a 1 , com as eficientes recebem uma pontuação de 1. Os modelos DEA podem ser orientados para inputs ou para outputs, bem como pode ser orientado como retornos constantes de escala (CRS) ou retornos variáveis de escala (VRS).

Modelos orientados para output procuram maximizar a produção para uma determinada quantidade de fatores de entrada. Por outro lado, orientada para inputs buscam minimizar os fatores de entrada necessários para um determinado nível de produção. Os modelos orientados para inputs são apropriados para o setor de distribuição de energia elétrica, uma vez que a demanda por serviços de distribuição é uma demanda relevante fora 
do controle das entidades, mas que deve de ser atendida. O presente estudo adota apenas modelos CRS, orientados para inputs.

$\mathrm{O}$ cálculo do escore de eficiência da i-ésima empresa em uma amostra de $\mathrm{N}$ entidades em modelos CRS pode ser especificado conforme a equação 1 abaixo:

$$
\begin{gathered}
\min _{\theta \lambda} \theta \\
\text { sujeito } a: \\
-y_{i}+Y \lambda \geq 0 \\
\theta x_{i}-X \lambda \geq 0 \\
\lambda \geq 0
\end{gathered}
$$

Onde $\theta$ é um escalar (escore de eficiência) e $\lambda$ representa um vetor Nx1 de constantes. Assumindo que as entidades utilizam $\mathrm{E}$ inputs e $\mathrm{M}$ outputs, $\mathrm{X}$ e $\mathrm{Y}$ representam a matriz de inputs $\mathrm{E} \times \mathrm{N}$ e a matriz de output $\mathrm{M} \times \mathrm{N}$, respectivamente.

O método DEA é o mais comumente utilizado na prática de benchmarking no setor de energia elétrica (JAMASB; POLLITT, 2003). Isto pode ser explicado pela relativa simplicidade dos modelos DEA e a possibilidade da sua implementação em um pequeno conjunto de dados. Cabe ressaltar que cada abordagem existe vários modelos que pode ser utilizado e a escolha do modelo não é simples. Vários estudos relataram discrepâncias nas estimativas de eficiência entre diferentes abordagens e especificações do modelo (ESTACHE; ROSSI; RUZZIER, 2004; FARSI; FILIPPINI; GREENE, 2006; JAMASB; POLLITT, 2003).

Visando determinar a eficiência no desempenho econômico-financeiro de cada concessionária, inicialmente foi encontrado um score de eficiência para cada entidade, com base no modelo DEA-CRS, em cada período de análise. Definidos os scores, cada entidade foi classificada com base nos grupos identificados pelo tipo de capital $(\mathrm{GPi}=$ privado e $\mathrm{GPu}=$ público).

Em seguida, foi aplicado o Teste Mann-Whitney-Wilcoxon (MWW), por ano, a partir do score gerado pelos grupos. Existe uma extensa literatura que compara testes paramétricos (o mais conhecido é teste $t$ ) com o teste MWW. O teste $t$, por exemplo, tem mais robustez para distribuições normais com variâncias iguais (BLAIR; HIGGINS, 1980; CRIBBIE; KESELMAN, 2003; DE WINTER; DODOU, 2010; RASCH; GUIARD， 2004; ZIMMERMAN; ZUMBO, 1993). Para distribuições altamente não normais, por outro lado, tais como exponencial, logarítma, ou com presença de outliers, o teste MWW tem mais robustez.

Há também uma preferência ao uso de teste não paramétricos em relação ao efeito tamanho da amostra. Conforme Lumley et al. (2002), muitos livros-texto e artigos mencionam que testes não paramétricos são preferíveis quando o tamanho da amostra é pequeno e o teste $t$ torna-se superior quando o tamanho da amostra aumenta.

Assim, para o cálculo da soma dos ranks, considerou-se:

(a) a posição de cada entidade, se eficiência menor que 1;

(b) o resultado da equação $\frac{\Sigma\left(1+2+\ldots+n_{e}\right)}{n_{e}}$, se a eficiência igual a 1 , onde $n_{e}$ é o número de entidades eficientes (com eficiência igual a 1). 
Para o cálculo dos índices U-values foram utilizadas a seguintes equações 2 e 3 :

$$
\begin{aligned}
U_{G P i} & =n_{i} n_{u}+0,5 \times\left(n_{i}\right) \times\left(n_{i}+1\right)-S_{i} \\
U_{G P_{u}} & =n_{i} n_{u}+0,5 \times\left(n_{u}\right) \times\left(n_{u}+1\right)-S_{u}
\end{aligned}
$$

onde:

$U_{G P i}, U_{G P u}=U$-values para os grupos de entidades privadas e públicas, respectivamente.

$n_{i}, n_{u}=$ tamanho dos grupos de entidades privadas e públicas, respectivamente.

$S_{i}, S_{u}=$ soma dos ranks dos grupos de entidades privadas e públicas, respectivamente.

O Teste MWW soma os postos pelo ranking das observações dos grupos, em conjunto, em ordem crescente. Os dois grupos não precisam ter o mesmo tamanho, mais amostras razoavelmente grandes possuem resultados, como resultado do Teorema do Limite Central.

\section{RESULTADOS E ANÁLISES}

Esta seção descreve a análise e a interpretação dos resultados encontrados, com base no score de eficiência das concessionárias de distribuição de energia elétrica. Com isso, partiu-se para um comparativo entre as concessionárias privadas e públicas.

A Tabela 2 mostra o resumo da estatística para as variáveis selecionadas no estudo.

Tabela 2 - Estatística descritiva das variáveis selecionadas

\begin{tabular}{cccccc}
\hline Variáveis & Unidade & Média & Desvio-padrão & Mínimo & Máximo \\
\hline OPEX & R \$ milhões & 343,68 & 481,69 & 1 & 2.904 \\
CL & Milhares & 1.148 & 1.598 & 3 & 7.782 \\
ED & GWh & 5.000 & 7.417 & 12 & 37.874 \\
DR & $\mathrm{Km} / \mathrm{Km}^{2}$ & 0,9738 & 0,8678 & 0,0099 & 4,7059 \\
\hline
\end{tabular}

Fonte: Dados de pesquisa.

Em seguida, a Tabela 3 foi elaborada para sumarizar a eficiência das entidades analisadas em cada um dos períodos.

Tabela 3 - Estatística descritiva dos scores de eficiências

\begin{tabular}{lccccc}
\hline Grupos & 2014 & 2013 & 2012 & 2011 & 2010 \\
\hline GPi & & & & & \\
$\quad N^{o}$ de entidades & 45 & 45 & 45 & 45 & 45 \\
$\quad$ Média & 0,6566 & 0,6566 & 0,6329 & 0,6762 & 0,6255 \\
$\quad$ Desvio-padrão & 0,1651 & 0,1870 & 0,1809 & 0,2031 & 0,2051 \\
$\quad$ Mínimo & 0,3202 & 0,2892 & 0,2686 & 0,1419 & 0,1202 \\
$\quad$ Máximo & 1,0000 & 1,0000 & 1,0000 & 1,0000 & 1,0000 \\
$\mathrm{GPu}$ & & & & & \\
$\quad N^{o}$ de entidades & 17 & 17 & 17 & 17 & 17
\end{tabular}


Revista Ambiente Contábil - ISSN 2176-9036 - UFRN - Natal-RN. v. 8. n. 2, p. 263 - 286, jul./dez. 2016.

\begin{tabular}{llllll} 
Média & 0,4518 & 0,4417 & 0,4772 & 0,4988 & 0,4367 \\
Desvio-padrão & 0,1963 & 0,2045 & 0,2450 & 0,2182 & 0,1818 \\
Mínimo & 0,1574 & 0,1238 & 0,1172 & 0,1271 & 0,1027 \\
Máximo & 1,0000 & 0,9056 & 1,0000 & 1,0000 & 0,9122 \\
\hline
\end{tabular}

Fonte: Dados de pesquisa.

Na Tabela 3, cada período de análise é demonstrado em uma coluna, constando as principais estatísticas descritivas. O modelo CRS considera retornos constantes de escala dado um conjunto de $I$ inputs e $O$ outputs. O score varia de 0 a 1 . Quanto maior o score, mais se aproxima da fronteira de eficiência. O score igual a 1 significa que a entidade é eficiente, ou seja, atingiu o ponto máximo da fronteira, formado pelos índices de eficiências encontradas as demais entidades. Por outro lado, os menores scores remetem às entidades com menor eficiência entre o grupo analisado, pois são as mais distantes da fronteira.

O Anexo I mostra os scores individuais por período. Neste sentido, destacam-se como eficientes entre as privadas, CPFL Jaguarari e Muxenergia (2010 a 2014), Sulgipe (2012 a 2014), Coelce (2011), Coelba e CPFL Paulista (2010). Entre as públicas, as eficientes são Celesc (2011 e 2014) e Celg (2012).

Na outra ponta, as menos eficientes entre as privadas são: a Empresa Força e Luz João Cesa Ltda - EFLJC (0,3202 em 2014; 0,2892 em 2013 e 0,2686 em 2012) e Caiuá (0,1419 em 2011 e 0,1202 em 2010). Entre as públicas, a menos eficiente é a Amazonas em todos os períodos $(0,1574$ em 2014; 0,1238 em 2013; 0,1172 em 2012; 0,1271 em 2011 e 0,1027 em 2010).

Vale destacar que a mediana dos scores das entidades privadas é superior que a das entidades públicas em todos os períodos. O Teste Mann-Whitney-Wilcoxon é utilizado para calcular, com base nos ranks de cada entidade que compõem a amostra, se a diferença entre as medianas dos scores dos grupos é estatisticamente significativa.

A Tabela 4 apresenta os resultados do teste de normalidade conforme o Teste de Jarque-Bera para os scores de eficiências.

Tabela 4 - Resultados do Teste de normalidade de Jarque-Bera

\begin{tabular}{lccccc}
\hline Descrição & 2014 & 2013 & 2012 & 2011 & 2010 \\
\hline Estatística & 0,2193 & 0,9493 & 0,9846 & 0,6526 & 1,0051 \\
P-valor & 0,8961 & 0,6221 & 0,6112 & 0,7216 & 0,6050
\end{tabular}

Fonte: Dados de pesquisa.

O Teste de Jarque-Bera tem como hipótese nula a normalidade. Assim, se o p-valor for menor que o nível crítico estabelecido ( $\mathrm{p}<5 \%$, por exemplo), então rejeita-se a normalidade dos scores de eficiências. Já se $\mathrm{p}>5 \%$, não se rejeita a hipótese nula. Os resultados sugerem que, no período considerado no estudo, os scores se comportam em distribuição normal ao nível crítico de $5 \%$.

A Tabela 5 retrata os resultados do Teste MWW em cada um dos períodos. 
Tabela 5 - Resultados do Teste Mann-Whitney-Wilcoxon

\begin{tabular}{|c|c|c|c|c|c|}
\hline Grupos & 2014 & 2013 & 2012 & 2011 & 2010 \\
\hline \multicolumn{6}{|l|}{ Soma dos Ranks } \\
\hline$G P i$ & $1.665,50$ & $1.633,00$ & $1.594,50$ & $1.588,50$ & $1.630,00$ \\
\hline$G P u$ & 287,50 & 320,00 & 358,50 & 364,50 & 323,00 \\
\hline $\begin{array}{l}\text { W (soma dos } \\
\text { postos, } G P u)\end{array}$ & 287,50 & 320,00 & 358,50 & 364,50 & 323,00 \\
\hline $\mathrm{Z}$ & $-3,9133$ & $-3,4005$ & $-2,7930$ & $-2,6983$ & $-3,3531$ \\
\hline Prob. $(\mathrm{Z}<\mathrm{z})$ & 0,000046 & 0,000336 & 0,002612 & 0,003485 & 0,000400 \\
\hline P-valor bicaudal & 0,000091 & 0,000673 & 0,005223 & 0,006970 & 0,000799 \\
\hline
\end{tabular}

Fonte: Dados de pesquisa.

Na Tabela 5, as primeiras duas linhas contemplam a soma dos ranks de cada entidade analisada nesta pesquisa, por período. Mais detalhadamente, o Anexo II mostra os ranks individuais, ordenados de acordo com o score gerado, partindo do menor para o maior. $\mathrm{O}$ score varia de 1 a 62 de acordo com a posição na amostra. $\mathrm{O}$ score igual a 1 significa que a entidade tem a posição $\mathrm{n}^{\circ} 1$ (mais eficiente) enquanto o score igual a 62 significa que a entidade tem a posição $n^{\circ} 62$ (menos eficiente).

$\mathrm{Na}$ terceira linha, são apresentados os índices $W$ a partir da soma dos ranks do grupo das entidades públicas $(G P u)$. Nas demais linhas, apresentam a estatística $z$. Se estatística $z$ calculada for menor que $\mathrm{Z}$ crítico, significa que as medianas dos scores dos grupos são estatisticamente diferentes entre si.

Desse modo, em todos os períodos analisados, as medianas dos grupos são diferentes entre si, ao nível de $1,0 \%$. A análise dos dados e o resultado do teste sugere uma interpretação que as entidades privadas possuem uma mediana diferente as entidades públicas. Alguns estudos corroboram com esses achados dessa pesquisa como Bagdadioglu, Price e WeymanJones (1996) e Kumbahkar e Hjalmarsson (1996).

Mesmo assim, a natureza da ocorrência dos dados requer o uso de controles para que os resultados das comparações sejam confiáveis, e testes de robustez adicionais são necessários. Para tanto, formulou-se dois testes de robustez que exploram mais diretamente a diferença de scores entre as entidades: a tendência temporal e a inclusão de variáveis de controle (porte da entidade e região de atuação).

No primeiro teste, considera-se avaliar a persistência da diferença em relação ao tempo. A ideia é incluir a iteração dos scores de eficiência, uma variável dummy para presença do governo $(0=$ não e $1=\operatorname{sim})$, e variáveis para cada período analisado (dummyl4 para ano 2014, dummyl3 para ano 2013, e assim por diante). Como os dados estão disponíveis em cross-section para cada período de tempo, as variações dummy por ano indica as iterações destas com as entidades analisadas.

Se os coeficientes estimados destas iterações forem estatisticamente significativos, então as diferenças entre as entidades persistem com o passar dos anos, com sinal positivo, se há tendência de aumento da diferença, ou negativo, caso haja tendência de queda.

Os resultados desta regressão encontram-se na Tabela 6 a seguir. 
Revista Ambiente Contábil - ISSN 2176-9036 - UFRN - Natal-RN. v. 8. n. 2, p. 263 - 286, jul./dez. 2016.

Tabela 6 - Resultados da regressão eficiência $\mathrm{x}$ governo $\mathrm{x}$ ano

\begin{tabular}{lcccc}
\hline \multicolumn{1}{c}{ Variáveis } & coeficiente & erro padrão & razão-t & p-valor \\
\hline constante & 0,625379 & 0,0255148 & 24,51 & 0,0001 *** \\
Governo & $-0,188347$ & 0,0246633 & $-7,637$ & 0,0000 *** \\
dummy14 & 0,0267245 & 0,0347929 & 0,7681 & 0,4430 \\
dummy13 & 0,0239108 & 0,0347929 & 0,6872 & 0,4925 \\
dummy12 & 0,0164708 & 0,0347929 & 0,4734 & 0,6363 \\
dummy11 & 0,0538071 & 0,0347929 & 1,5460 & 0,1230 \\
\hline
\end{tabular}

Fonte: Dados de pesquisa.

$* * *$ significativo a $1 \%$.

A variável dummy10 referente ao ano 2010 foi omitida devido a colinearidade exata e não é significativa. Pode-se perceber que nenhuma das variáveis dummy associadas ao tempo são estatisticamente significativas. Isso implica não rejeita a hipótese nula de que a tendência temporal exerça influência nos scores de eficiência.

O segundo teste considera a inclusão de variáveis de controle. Foram selecionadas as variáveis porte da entidade e região de atuação. O Anexo III mostra as características das entidades conforme o porte e a região de atuação. Os resultados das regressões considerando a inclusão dessa variável são mostrados na Tabelas 7 a seguir.

A variável porte da entidade compreende o efeito que o tamanho da própria entidade exerce sobre a eficiência. Para fins de operacionalização da pesquisa, as entidades, privadas e públicas, foram divididas em dois grupos, conforme a quantidade de energia distribuída: abaixo de $5.000 \mathrm{GWh}($ dummy $=0)$ e acima de $5.000 \mathrm{GWh}($ dummy $=1)$.

Tabela 7 - Resultados da regressão eficiência $\mathrm{x}$ governo $\mathrm{x}$ porte

\begin{tabular}{lcccc}
\hline \multicolumn{1}{c}{ Variáveis } & coeficiente & erro padrão & razão-t & p-valor \\
\hline Constante & 0,594538 & 0,0137573 & 43,22 & $0,0000 * * *$ \\
Governo & $-0,191584$ & 0,0225311 & $-8,503$ & $0,0000 * * *$ \\
Porte & 0,165071 & 0,0212379 & 7,772 & $0,0000 * * *$ \\
\hline
\end{tabular}

Fonte: Dados de pesquisa.

*** significativo a $1 \%$.

Note-se que a variável porte é estatisticamente significativa, ou seja, o porte exerce influência nos scores de eficiência. A Tabela 8 sumariza os scores de eficiência das entidades, divididas conforme o porte. 
Tabela 8 - Estatística descritiva dos scores de eficiências conforme o porte

\begin{tabular}{lcccccc}
\hline Grupos & & 2014 & 2013 & 2012 & 2011 & 2010 \\
\hline & GPi & & & & & \\
& $N^{o}$ de entidades & 15 & 16 & 15 & 15 & 14 \\
Acima de & Média & 0,7064 & 0,7129 & 0,7069 & 0,7861 & 0,7548 \\
$5.000 \mathrm{GWh}$ & $\mathrm{GPu}$ & & & & & \\
& $N^{o}$ de entidades & 6 & 6 & 6 & 6 & 6 \\
& Média & 0,6137 & 0,6301 & 0,7023 & 0,6609 & 0,5676 \\
\hline & $\mathrm{GPi}$ & & & & & \\
& $N^{o}$ de entidades & 30 & 29 & 30 & 30 & 31 \\
Abaixo de & Média & 0,6317 & 0,6255 & 0,5959 & 0,6212 & 0,5671 \\
$5.000 \mathrm{GWh}$ & $\mathrm{GPu}$ & & & & & \\
& $N^{o}$ de entidades & 11 & 11 & 11 & 11 & 11 \\
& Média & 0,3635 & 0,3389 & 0,3544 & 0,4103 & 0,3653 \\
\hline
\end{tabular}

Fonte: Dados de pesquisa.

A variável região de atuação compreende o efeito que a localidade exerce sobre a eficiência. Para fins de operacionalização da pesquisa, foram criadas cinco variáveis dummy, uma para cada região $(\mathrm{regN}=$ norte, regNE = nordeste, regCO = centro-oeste, regSE $=$ sudeste e regS = sul). Os resultados das regressões considerando a inclusão dessa variável são mostrados na Tabelas 9 a seguir.

Tabela 9 - Resultados da regressão eficiência x governo x região

\begin{tabular}{lcccc}
\hline \multicolumn{1}{c}{ Variáveis } & coeficiente & erro padrão & razão-t & p-valor \\
\hline constante & 0,556558 & 0,0347945 & 16,00 & $0,0000 * * *$ \\
Governo & $-0,101483$ & 0,0237126 & $-4,280$ & $0,0000 * * *$ \\
regN & $-0,171949$ & 0,0444603 & $-3,867$ & $0,0001 * * *$ \\
regNE & 0,216068 & 0,0407045 & 5,308 & $0,0000 * * *$ \\
regSE & 0,098520 & 0,0378036 & 2,606 & $0,0096 * * *$ \\
regS & 0,055822 & 0,0380992 & 1,465 & 0,1439 \\
\hline
\end{tabular}

Fonte: Dados de pesquisa.

*** significativo a $1 \%$.

A variável regCO referente à região centro-oeste foi omitida devido a colinearidade exata e não é significativa. Apenas as variáveis regN, regNE e regSE são estatisticamente significativas. Contudo, o coeficiente da variável regN é negativo, o que implica uma influência negativa sobre os scores de eficiência. Isso se explica porque a região possui sistemas isolados, que respondem pelo fornecimento de energia em áreas de difícil acesso e de baixa densidade populacional. 

região.

A Tabela 10 sumariza os scores de eficiência das entidades, divididas conforme a

Tabela 10 - Estatística descritiva dos scores de eficiências conforme a região

\begin{tabular}{|c|c|c|c|c|c|c|}
\hline Grupos & & 2014 & 2013 & 2012 & 2011 & 2010 \\
\hline \multirow{6}{*}{ Norte } & GPi & & & & & \\
\hline & $N^{o}$ de entidades & 2 & 2 & 2 & 2 & 2 \\
\hline & Média & 0,4535 & 0,5074 & 0,4449 & 0,5317 & 0,4338 \\
\hline & $\mathrm{GPu}$ & & & & & \\
\hline & $N^{o}$ de entidades & 5 & 5 & 5 & 5 & 5 \\
\hline & Média & 0,2657 & 0,2109 & 0,2563 & 0,2524 & 0,2512 \\
\hline \multirow{6}{*}{ Nordeste } & GPi & & & & & \\
\hline & $N^{o}$ de entidades & 9 & 9 & 9 & 9 & 9 \\
\hline & Média & 0,7839 & 0,8446 & 0,7774 & 0,8676 & 0,7649 \\
\hline & $\mathrm{GPu}$ & & & & & \\
\hline & $N^{o}$ de entidades & 2 & 2 & 2 & 2 & 2 \\
\hline & Média & 0,5073 & 0,4558 & 0,4765 & 0,6244 & 0,5032 \\
\hline \multirow{6}{*}{ Centro-Oeste } & GPi & & & & & \\
\hline & $N^{o}$ de entidades & 3 & 3 & 3 & 3 & 3 \\
\hline & Média & 0,4824 & 0,4497 & 0,4562 & 0,5157 & 0,4528 \\
\hline & $\mathrm{GPu}$ & & & & & \\
\hline & $N^{o}$ de entidades & 2 & 2 & 2 & 2 & 2 \\
\hline & Média & 0,5369 & 0,6339 & 0,7772 & 0,4998 & 0,4666 \\
\hline \multirow{6}{*}{ Sudeste } & GPi & & & & & \\
\hline & $N^{o}$ de entidades & 20 & 20 & 20 & 20 & 20 \\
\hline & Média & 0,6867 & 0,6793 & 0,6564 & 0,6760 & 0,6425 \\
\hline & $\mathrm{GPu}$ & & & & & \\
\hline & $N^{o}$ de entidades & 2 & 2 & 2 & 2 & 2 \\
\hline & Média & 0,3687 & 0,4140 & 0,3892 & 0,5088 & 0,4315 \\
\hline \multirow{6}{*}{ Sul } & GPi & & & & & \\
\hline & $N^{o}$ de entidades & 11 & 11 & 11 & 11 & 11 \\
\hline & Média & 0,5823 & 0,5449 & 0,5543 & 0,5900 & 0,5625 \\
\hline & $\mathrm{GPu}$ & & & & & \\
\hline & $N^{o}$ de entidades & 6 & 6 & 6 & 6 & 6 \\
\hline & Média & 0,5878 & 0,5744 & 0,5907 & 0,6586 & 0,5608 \\
\hline
\end{tabular}

Fonte: Dados de pesquisa.

A presente pesquisa foi realizada com as 62 concessionárias brasileiras de distribuição de energia elétrica. A eficiência no desempenho econômico-financeiro dessas entidades 
depende, sobretudo, da gestão eficiente dos recursos, que refletem diretamente nos índices que determinam sua eficiência.

\section{CONSIDERAÇÕES FINAIS}

Com vistas a verificar a eficiência das concessionárias de distribuição de energia elétrica, adotou-se o modelo DEA-CRS para determinar um score a partir das variáveis selecionadas na pesquisa. O score gerado não determina, em termos absolutos, o quanto eficiente é cada entidade, mas permite identificar a eficiência relativa em relação ao conjunto de entidades da amostra.

Em relação à questão de pesquisa, a análise dos dados e o resultado do teste sugere uma interpretação que as entidades privadas possuem mediana dos scores de eficiência significativamente diferente das entidades públicas. Os resultados, porém, devem ser interpretados com cautela à luz das variáveis de controle como porte e região de atuação.

Logicamente, os resultados são limitados apenas para a amostra analisada, de modo que não pode ser generalizado às demais entidades fora do contexto brasileiro.

O score de eficiência é determinado a partir dos custos operacionais, densidade da rede, número de clientes e energia distribuída. Estes fatores, em conjunto, podem estar contribuindo para um melhor (ou pior) desempenho econômico-financeiro entre as entidades, principalmente os custos.

As entidades podem melhorar os índices de eficiência através de uma gestão mais eficiente dos recursos, que afetam os custos do serviço. Por conseguinte, a melhoria do desempenho maximiza o retorno à entidade, ao menos até a próxima revisão tarifária.

Recomenda-se analisar o desempenho de entidades privadas e públicas estrangeiras e compará-las com as brasileiras. Partindo da premissa que os países possuem um Poder Público atuante, é razoável afirmar que há interferências deste no setor elétrico, seja atuando como empresa ou como órgão regulador.

Em virtude dos resultados aqui apresentados, adstritos aos aspectos econômicofinanceiros, recomenda-se a inclusão de outras variáveis para identificar outras influências e controles dos resultados obtidos, tais como formas de organização jurídica das entidades, concentração de capital do acionista majoritário, etc.

Por fim, a contribuição deste estudo se endereça também ao órgão regulador, a Aneel. Pelo lado do consumidor, a diferença entre entidades privadas e públicas não é contemplada no modelo regulatório; contudo, propõe-se aperfeiçoar os mecanismos que norteiam os reajustes das tarifas para o consumidor levando em consideração as diferenças entre as entidades, buscando um equilíbrio entre os custos assumidos pelos consumidores, a necessidade de investimentos e a modicidade tarifária.

\section{REFERÊNCIAS}

ABBOTT, M. Determining levels of productivity and efficiency in the Electricity Industry. The Electricity Journal, 18(9), 62-72, 2005

AROCENA, P.; PRICE, C. W. Generating efficiency: economic and environmental regulation of public and private electricity generators in Spain. International Journal of Industrial Organization, 20, 41-69, 2002

BAGDADIOGLU, N.; PRICE, C. W.; WEYMAN-JONES, T. G. Efficiency and ownership in electricity distribuition: a non-parametric model of the Turkish experience. Energy Economics, 18, 1-23, 1996 
BAUMOL, W.; WILLING, R. D. Fixed cost, sunk costs, entry barriers, and sustainability of monopoly. Quarterly Journal of Economics, 96 (3), 405-431, 1981

BLAIR, R. C.; HIGGINS, J. J. A comparison of the power of Wilcoxon's rank-sum statistic to that of Student's $t$ statistic under various non normal distributions. Journal of Educational Statistics, 5, 309-335, 1980

BRASIL. Agência nacional de energia elétrica - ANEEL. Disponível em: <http://www.aneel.gov.br>. Acesso em: 31 mar. 2015.

BRASIL. ANEEL. Agência Nacional de Energia Elétrica. Resolução ANEEL nº 396, de 23 de fevereiro de 2010. Institui a Contabilidade Regulatória e aprova alterações no Manual de Contabilidade do Setor Elétrico, instituído pela Resolução ANEEL n 444, de 26 de outubro de 2001

BRASIL. ANEEL. Agência Nacional de Energia Elétrica. Resolução ANEEL nº 457, de 9 de novembro de 2011. Aprova o Módulo 2 dos Procedimentos de Regulação Tarifária PRORET, o qual define a metodologia e os procedimentos gerais para realização do Terceiro Ciclo de Revisões Tarifárias Periódicas das Concessionárias de Distribuição de Energia Elétrica - 3CRTP

BRASIL. Lei $\mathrm{n}^{\circ}$ 9.427, de 26 de dezembro de 1996. Institui a Agência Nacional de Energia Elétrica - ANEEL, disciplina o regime das concessões de serviços públicos de energia elétrica e dá outras providências. Diário Oficial [da] República Federativa do Brasil. Brasília - DF Disponível em: <http://www.planalto.gov.br/ccivil_03/leis/L9427cons.htm>. Acesso em: 31 mar. 2015.

BRASIL. Lei $\mathrm{n}^{\circ} 10.848$, de 15 de março de 2004. Dispõe sobre a comercialização de energia elétrica, altera as Leis $\mathrm{n}^{\circ}$. 5.655, de 20 de maio de 1971, 8.631, de 4 de março de 1993, 9.074, de 7 de julho de 1995, 9.427, de 26 de dezembro de 1996, 9.478, de 6 de agosto de 1997, 9.648, de 27 de maio de 1998, 9.991, de 24 de julho de 2000, 10.438, de 26 de abril de 2002, e dá outras providências. Diário Oficial [da] República Federativa do Brasil. Brasília - DF. Disponível em: <http://www.planalto.gov.br/ccivil_03/_ato2004-2006/2004/lei/110.848.htm>. Acesso em: 31 mar. 2015.

BUBICZ, M. E.; PEREIRA, G. M.; BORCHARDT, M.; SELLITTO, M. A. Ganhos de empresas industriais brasileiras com o mercado livre de energia elétrica. GEINTEC. 4(1), 588-603, 2014

CRIBBIE, R. A.; KESELMAN, H. J. The effects of nonnormality on parametric, nonparametric, and model comparison approaches to pairwise comparisons. Educational and Psychological Measurement, 63, 615-635, 2003

DE WINTER, J. C. F.; DODOU, D. Five-point Likert items: t test versus Mann-WhitneyWilcoxon. Practical Assessment, Research \& Evaluation, 15, 11, 2010

DEMSETZ, H. Why regulate utilities? Journal of Law and Economics, 55-65, 1968 
ESTACHE, A.; ROSSI, M. A.; RUZZIER, C. A. The case for international coordination of electricity regulation: evidence from the Measurement of Efficiency in South America. Journal of Regulatory Economics, 25(3), 271-295, 2004

FARRELL, M. J. The measurement of productive efficiency. Journal of the Royal Statistical Society. Series A (3), 253-290, 1957

FARSI, M.; FILIPPINI, M.; GREENE, W. H. Application of panel data models in benchmarking analysis of the electricity distribution sector. In: Annals of Public and Cooperative Economics, 77(3), 271-290, 2006

HANKE, S. Privatization. In: The New Palgrave Dictionary of Economics. Basil Blackwell, v. 4, 1991

JAMASB, T. J.; POLLITT, M. G. International benchmarking and regulation: an application to European electricity distribution utilities. Energy Policy, 31(15), 1609-1622, 2003

JARREL, G. The demand for state regulation of the electric utility industry. Journal of Law and Economics, 21(2), 269-296, 1978

KESSLER, M. R. A regulação econômica no setor elétrico brasileiro: teoria e evidências. Dissertação (Mestrado Profissional em Economia) - Universidade Federal do Rio Grande do Sul, Faculdade de Ciências Econômicas, Programa de Pós-Graduação em Economia, Porto Alegre, 2006. 169 p.

KOH, D. S.; BERG, S.; KENNY, L. W. A Comparison of costs in privately-owned and publicly-owned electric utilities: the role of scale. Land Economics, 1995

KUMBHAKAR, S. C.; HJALMARSSON, L. Relative performance of public and private ownership under yardstick competition: electricity retail distribution. European Economic Review, 42, 97-122, 1998

LIMA, E. C. P. Empresas estatais, retorno de investimento e ajuste fiscal: a privatização é um bom negócio para o governo? Brasília: ESAF, 1997, 58 p.

LUMLEY T; DIEHR P; EMERSON S; CHEN L. The importance of the normality assumption in large public health data sets. Annual Review of Public Health, 23, 151-169, 2002

MEGginson, W. L. The Financial Economics of Privatization. New York: Oxford University Press, 2005

RASCH, D.; GUIARD, V. The robustness of parametric statistical methods. Psychology Science, 46, 175-208, 2004

RUDNICK, H.; ZOLEZZI, J. Electric sector deregulation and restructuring in Latin America: lessons to be learnt and possible ways forward. IEE, 148(2), 180-184, 2001

SAMPAIO, L. M. B; RAMOS, F. S.; SAMPAIO, Y. Privatização e eficiência das usinas hidrelétricas brasileiras. Econ. Aplic, 9(3): 465-480, 2005 
SHAPIRO, C.; WILLIG, R. Economic Rationales for the Scope of Privatization. In: SUlEIMAN, B. N.; WATERBURY, J. (Ed.). The Political Economy of Public Sector and Privatization. London: Westview, 1990

SHLEIFER, A. A theory of yardstick competition. Rand Journal of Economics, 16(3), 319327,1985

VICKERS, J.; YARROW, G.. Privatization: An Economic Analysis. Cambridge: MIT, 1988

YARROW. G. Privatization in theory and practice. Economic Policy, 2, 1986

ZIMMERMAN, D. W.; ZUMBO, B. D. The relative power of parametric and nonparametric statistical methods. In G. Keren \& C. Lewis (Eds.), A handbook for data analysis in the behavioral sciences: Methodological issues (pp. 481-517). Hillsdale, NJ: Erlbaum. 1993 
Anexo I

Scores individuais por período analisado

\begin{tabular}{|c|c|c|c|c|c|c|}
\hline Entidades & Grupo & 2014 & 2013 & 2012 & 2011 & 2010 \\
\hline AES-SUL & Privado & 0,7068 & 0,6941 & 0,7338 & 0,7401 & 0,8034 \\
\hline AMAZONAS & Público & 0,1574 & 0,1238 & 0,1172 & 0,1271 & 0,1027 \\
\hline AMPLA & Privado & 0,6004 & 0,6505 & 0,6173 & 0,7443 & 0,5848 \\
\hline BANDEIRANTE & Privado & 0,7969 & 0,7511 & 0,8502 & 0,8478 & 0,8382 \\
\hline BOA VISTA & Público & 0,2332 & 0,2039 & 0,2872 & 0,2696 & 0,2795 \\
\hline CAIUÁ-D & Privado & 0,6629 & 0,6670 & 0,4648 & 0,1419 & 0,1202 \\
\hline CELG-D & Público & 0,5310 & 0,7792 & 1,0000 & 0,5695 & 0,4043 \\
\hline CEMIG-D & Público & 0,4998 & 0,5221 & 0,4982 & 0,6109 & 0,4809 \\
\hline ELETROCAR & Público & 0,4247 & 0,4536 & 0,3322 & 0,4317 & 0,3634 \\
\hline CERON & Público & 0,3532 & 0,2028 & 0,3190 & 0,3238 & 0,2888 \\
\hline CELPA & Privado & 0,5363 & 0,5939 & 0,4948 & 0,5507 & 0,4600 \\
\hline COCEL & Público & 0,5577 & 0,5283 & 0,5834 & 0,6206 & 0,5689 \\
\hline CELPE & Privado & 0,6492 & 0,7728 & 0,7697 & 0,9841 & 0,7777 \\
\hline COELCE & Privado & 0,8769 & 0,9203 & 0,8722 & 1,0000 & 0,7714 \\
\hline CEMAR & Privado & 0,7271 & 0,8829 & 0,8121 & 0,8648 & 0,8129 \\
\hline CEPISA & Público & 0,5949 & 0,4244 & 0,4300 & 0,6872 & 0,5265 \\
\hline COSERN & Privado & 0,7690 & 0,8890 & 0,8370 & 0,9792 & 0,9224 \\
\hline CEEE-D & Público & 0,5324 & 0,5534 & 0,7120 & 0,7911 & 0,5467 \\
\hline CFLO & Privado & 0,6622 & 0,8102 & 0,5479 & 0,6369 & 0,5128 \\
\hline CHESP & Privado & 0,4796 & 0,4677 & 0,4777 & 0,5084 & 0,4302 \\
\hline CPFL JAGUARI & Privado & 1,0000 & 1,0000 & 1,0000 & 1,0000 & 1,0000 \\
\hline CPFL LESTE PAULISTA & Privado & 0,5817 & 0,5624 & 0,5640 & 0,5591 & 0,5470 \\
\hline CPFL MOCOCA & Privado & 0,6283 & 0,6245 & 0,6598 & 0,6857 & 0,5994 \\
\hline DEMEI & Público & 0,4357 & 0,4736 & 0,4678 & 0,5442 & 0,4415 \\
\hline DMED & Público & 0,2376 & 0,3059 & 0,2803 & 0,4068 & 0,3821 \\
\hline ELEKTRO & Privado & 0,7289 & 0,6919 & 0,6932 & 0,7386 & 0,9140 \\
\hline ELETROPAULO & Privado & 0,7028 & 0,6952 & 0,6753 & 0,7165 & 0,6270 \\
\hline EDEVP & Privado & 0,7365 & 0,7690 & 0,5537 & 0,5766 & 0,4603 \\
\hline EEB & Privado & 0,6268 & 0,6012 & 0,5452 & 0,5410 & 0,4980 \\
\hline EFLJC & Privado & 0,3202 & 0,2892 & 0,2686 & 0,2901 & 0,4551 \\
\hline EFLUL & Privado & 0,5609 & 0,3389 & 0,3617 & 0,3703 & 0,4335 \\
\hline ELFSM & Privado & 0,5180 & 0,5028 & 0,5155 & 0,4863 & 0,5110 \\
\hline EBO & Privado & 0,8739 & 0,9222 & 0,8086 & 0,9126 & 0,7191 \\
\hline EMT & Privado & 0,4539 & 0,4457 & 0,4590 & 0,4968 & 0,4766 \\
\hline EMS & Privado & 0,5136 & 0,4357 & 0,4320 & 0,5418 & 0,4518 \\
\hline EMG & Privado & 0,6194 & 0,6284 & 0,5856 & 0,6746 & 0,5889 \\
\hline ENF & Privado & 0,5370 & 0,5166 & 0,4836 & 0,5149 & 0,4420 \\
\hline EPB & Privado & 0,8134 & 0,7885 & 0,6742 & 0,7886 & 0,6835 \\
\hline ESE & Privado & 0,6377 & 0,6766 & 0,6030 & 0,7210 & 0,6209 \\
\hline
\end{tabular}


Revista Ambiente Contábil - ISSN 2176-9036 - UFRN - Natal-RN. v. 8. n. 2, p. 263 - 286, jul./dez. 2016.

\begin{tabular}{lcccccc}
\hline \multicolumn{1}{c}{ Entidades } & Grupo & 2014 & 2013 & 2012 & 2011 & 2010 \\
\hline ETO & Privado & 0,3706 & 0,4208 & 0,3950 & 0,5128 & 0,4075 \\
ESCELSA & Privado & 0,6544 & 0,5667 & 0,5773 & 0,6246 & 0,5800 \\
FORCEL & Privado & 0,3922 & 0,3205 & 0,3872 & 0,3633 & 0,3126 \\
HIDROPAN & Privado & 0,4717 & 0,4041 & 0,4943 & 0,5961 & 0,5333 \\
IENERGIA & Privado & 0,4560 & 0,4089 & 0,4996 & 0,6584 & 0,5811 \\
LIGHT & Privado & 0,6281 & 0,6076 & 0,6771 & 0,7159 & 0,6644 \\
MUXENERGIA & Privado & 1,0000 & 1,0000 & 1,0000 & 1,0000 & 1,0000 \\
RGE & Privado & 0,7595 & 0,6951 & 0,7851 & 0,8305 & 0,6636 \\
UHENPAL & Privado & 0,5032 & 0,4741 & 0,4405 & 0,4575 & 0,3834 \\
\hline
\end{tabular}




\section{Anexo II}

Ranks individuais por período analisado

\begin{tabular}{|c|c|c|c|c|c|c|}
\hline Entidades & Grupo & 2014 & 2013 & 2012 & 2011 & 2010 \\
\hline AES-SUL & Privado & 17 & 21 & 15 & 18 & 11 \\
\hline AMAZONAS & Público & 62 & 62 & 62 & 62 & 62 \\
\hline AMPLA & Privado & 31 & 27 & 25 & 16 & 24 \\
\hline BANDEIRANTE & Privado & 10 & 16 & 8 & 12 & 9 \\
\hline BOA VISTA & Público & 61 & 60 & 58 & 59 & 59 \\
\hline CAIUÁ-D & Privado & 20 & 25 & 46 & 61 & 61 \\
\hline CEB-DIS & Público & 38 & 42 & 33 & 52 & 33 \\
\hline CELESC-DIS & Público & 1 & 6 & 5 & 1 & 8 \\
\hline CELG-D & Público & 42 & 13 & 1 & 36 & 53 \\
\hline CEMIG-D & Público & 46 & 39 & 40 & 32 & 40 \\
\hline ELETROCAR & Público & 52 & 47 & 56 & 51 & 56 \\
\hline CERON & Público & 56 & 61 & 57 & 56 & 58 \\
\hline CELPA & Privado & 40 & 32 & 41 & 40 & 44 \\
\hline COCEL & Público & 37 & 38 & 28 & 31 & 28 \\
\hline ELETROACRE & Público & 57 & 57 & 54 & 57 & 51 \\
\hline CEA & Público & 59 & 59 & 61 & 60 & 60 \\
\hline COELBA & Privado & 16 & 17 & 24 & 10 & 1 \\
\hline CEAL & Público & 53 & 43 & 37 & 38 & 41 \\
\hline CELPE & Privado & 23 & 14 & 14 & 5 & 12 \\
\hline COELCE & Privado & 6 & 5 & 7 & 1 & 13 \\
\hline CEMAR & Privado & 15 & 8 & 10 & 11 & 10 \\
\hline CEPISA & Público & 32 & 50 & 51 & 24 & 34 \\
\hline COSERN & Privado & 11 & 7 & 9 & 6 & 6 \\
\hline CEEE-D & Público & 41 & 36 & 16 & 14 & 30 \\
\hline CFLO & Privado & 21 & 11 & 35 & 29 & 36 \\
\hline CHESP & Privado & 47 & 46 & 44 & 47 & 50 \\
\hline CPFL JAGUARI & Privado & 1 & 1 & 1 & 1 & 1 \\
\hline CPFL LESTE PAULISTA & Privado & 33 & 34 & 32 & 39 & 29 \\
\hline CPFL MOCOCA & Privado & 26 & 29 & 22 & 25 & 22 \\
\hline CPFL SANTA CRUZ & Privado & 19 & 18 & 23 & 23 & 21 \\
\hline CNEE & Privado & 25 & 22 & 29 & 33 & 35 \\
\hline CPFL-PAULISTA & Privado & 8 & 10 & 12 & 8 & 1 \\
\hline CPFL- PIRATININGA & Privado & 5 & 9 & 6 & 7 & 5 \\
\hline CPFL SUL PAULISTA & Privado & 30 & 26 & 20 & 17 & 14 \\
\hline SULGIPE & Privado & 1 & 1 & 1 & 27 & 27 \\
\hline COOPERALIANÇA & Privado & 35 & 35 & 30 & 41 & 38 \\
\hline COPEL-DIS & Público & 34 & 37 & 48 & 37 & 32 \\
\hline DEMEI & Público & 51 & 45 & 45 & 42 & 48 \\
\hline DMED & Público & 60 & 56 & 59 & 53 & 55 \\
\hline ELEKTRO & Privado & 14 & 23 & 17 & 19 & 7 \\
\hline ELETROPAULO & Privado & 18 & 19 & 19 & 21 & 19 \\
\hline EDEVP & Privado & 13 & 15 & 34 & 35 & 43 \\
\hline EEB & Privado & 28 & 31 & 36 & 44 & 39 \\
\hline EFLJC & Privado & 58 & 58 & 60 & 58 & 45 \\
\hline EFLUL & Privado & 36 & 54 & 55 & 54 & 49 \\
\hline ELFSM & Privado & 43 & 41 & 38 & 49 & 37 \\
\hline EBO & Privado & 7 & 4 & 11 & 9 & 15 \\
\hline EMT & Privado & 50 & 48 & 47 & 48 & 42 \\
\hline EMS & Privado & 44 & 49 & 50 & 43 & 46 \\
\hline EMG & Privado & 29 & 28 & 27 & 26 & 23 \\
\hline ENF & Privado & 39 & 40 & 43 & 45 & 47 \\
\hline EPB & Privado & 9 & 12 & 21 & 15 & 16 \\
\hline ESE & Privado & 24 & 24 & 26 & 20 & 20 \\
\hline
\end{tabular}


Revista Ambiente Contábil - ISSN 2176-9036 - UFRN - Natal-RN. v. 8. n. 2, p. 263 - 286, jul./dez. 2016.

\begin{tabular}{|c|c|c|c|c|c|c|}
\hline Entidades & Grupo & 2014 & 2013 & 2012 & 2011 & 2010 \\
\hline ESCELSA & Privado & 22 & 33 & 31 & 30 & 26 \\
\hline HIDROPAN & Privado & 48 & 53 & 42 & 34 & 31 \\
\hline IENERGIA & Privado & 49 & 52 & 39 & 28 & 25 \\
\hline LIGHT & Privado & 27 & 30 & 18 & 22 & 17 \\
\hline UHENPAL & Privado & 45 & 44 & 49 & 50 & 54 \\
\hline
\end{tabular}




\section{Anexo III}

Porte e região de atuação

\begin{tabular}{|c|c|c|}
\hline Entidades & Porte & Região de Atuação \\
\hline AES-SUL & Grande (>5.000 GWh) & Sul \\
\hline AMAZONAS & Pequeno $(<5.000 \mathrm{GWh})$ & Norte \\
\hline AMPLA & Grande (>5.000 GWh) & Sudoeste \\
\hline BANDEIRANTE & Grande $(>5.000 \mathrm{GWh})$ & Sudoeste \\
\hline BOA VISTA & Pequeno $(<5.000 \mathrm{GWh})$ & Norte \\
\hline CAIUÁ-D & Pequeno $(<5.000 \mathrm{GWh})$ & Sudoeste \\
\hline CEB-DIS & Grande (>5.000 GWh) & Centro-Oeste \\
\hline CELESC-DIS & Grande $(>5.000 \mathrm{GWh})$ & Sul \\
\hline CELG-D & Grande (>5.000 GWh) & Centro-Oeste \\
\hline CEMIG-D & Grande (>5.000 GWh) & Sudoeste \\
\hline ELETROCAR & Pequeno $(<5.000 \mathrm{GWh})$ & Sul \\
\hline CERON & Pequeno $(<5.000 \mathrm{GWh})$ & Norte \\
\hline CELPA & Grande (>5.000 GWh) & Norte \\
\hline COCEL & Pequeno $(<5.000 \mathrm{GWh})$ & Sul \\
\hline ELETROACRE & Pequeno (<5.000 GWh) & Norte \\
\hline CEA & Pequeno $(<5.000 \mathrm{GWh})$ & Norte \\
\hline COELBA & Grande (>5.000 GWh) & Nordeste \\
\hline CEAL & Pequeno $(<5.000 \mathrm{GWh})$ & Nordeste \\
\hline CELPE & Grande $(>5.000 \mathrm{GWh})$ & Nordeste \\
\hline COELCE & Grande (>5.000 GWh) & Nordeste \\
\hline CEMAR & Pequeno $(<5.000 \mathrm{GWh})$ & Nordeste \\
\hline CEPISA & Pequeno $(<5.000 \mathrm{GWh})$ & Nordeste \\
\hline COSERN & Pequeno $(<5.000 \mathrm{GWh})$ & Nordeste \\
\hline CEEE-D & Grande (>5.000 GWh) & Sul \\
\hline CFLO & Pequeno $(<5.000 \mathrm{GWh})$ & Sul \\
\hline CHESP & Pequeno $(<5.000 \mathrm{GWh})$ & Centro-Oeste \\
\hline CPFL JAGUARI & Pequeno $(<5.000 \mathrm{GWh})$ & Sudoeste \\
\hline CPFL LESTE PAULISTA & Pequeno $(<5.000 \mathrm{GWh})$ & Sudoeste \\
\hline CPFL MOCOCA & Pequeno $(<5.000 \mathrm{GWh})$ & Sudoeste \\
\hline CPFL SANTA CRUZ & Pequeno $(<5.000 \mathrm{GWh})$ & Sudoeste \\
\hline CNEE & Pequeno $(<5.000 \mathrm{GWh})$ & Sudoeste \\
\hline CPFL-PAULISTA & Grande $(>5.000 \mathrm{GWh})$ & Sudoeste \\
\hline CPFL- PIRATININGA & Grande (>5.000 GWh) & Sudoeste \\
\hline CPFL SUL PAULISTA & Pequeno $(<5.000 \mathrm{GWh})$ & Sudoeste \\
\hline SULGIPE & Pequeno $(<5.000 \mathrm{GWh})$ & Nordeste \\
\hline COOPERALIANÇA & Pequeno $(<5.000 \mathrm{GWh})$ & Sul \\
\hline COPEL-DIS & Grande $(>5.000 \mathrm{GWh})$ & Sul \\
\hline DEMEI & Pequeno $(<5.000 \mathrm{GWh})$ & Sul \\
\hline DMED & Pequeno $(<5.000 \mathrm{GWh})$ & Sudoeste \\
\hline ELEKTRO & Grande (>5.000 GWh) & Sudoeste \\
\hline ELETROPAULO & Grande (>5.000 GWh) & Sudoeste \\
\hline EDEVP & Pequeno $(<5.000 \mathrm{GWh})$ & Sudoeste \\
\hline EEB & Pequeno $(<5.000 \mathrm{GWh})$ & Sudoeste \\
\hline EFLJC & Pequeno $(<5.000 \mathrm{GWh})$ & Sul \\
\hline EFLUL & Pequeno $(<5.000 \mathrm{GWh})$ & Sul \\
\hline ELFSM & Pequeno $(<5.000 \mathrm{GWh})$ & Sudoeste \\
\hline EBO & Pequeno $(<5.000 \mathrm{GWh})$ & Nordeste \\
\hline EMT & Grande (>5.000 GWh) & Centro-Oeste \\
\hline EMS & Pequeno $(<5.000 \mathrm{GWh})$ & Centro-Oeste \\
\hline EMG & Pequeno $(<5.000 \mathrm{GWh})$ & Sudoeste \\
\hline ENF & Pequeno (<5.000 GWh) & Sudoeste \\
\hline EPB & Pequeno $(<5.000 \mathrm{GWh})$ & Nordeste \\
\hline ESE & Pequeno $(<5.000 \mathrm{GWh})$ & Nordeste \\
\hline
\end{tabular}


Revista Ambiente Contábil - ISSN 2176-9036 - UFRN - Natal-RN. v. 8. n. 2, p. 263 - 286, jul./dez. 2016.

\begin{tabular}{lcc}
\hline \multicolumn{1}{c}{ Entidades } & Porte & Região de Atuação \\
\hline ETO & Pequeno $(<5.000 \mathrm{GWh})$ & Norte \\
ESCELSA & Grande $(>5.000 \mathrm{GWh})$ & Sudoeste \\
FORCEL & Pequeno $(<5.000 \mathrm{GWh})$ & Sul \\
HIDROPAN & Pequeno $(<5.000 \mathrm{GWh})$ & Sul \\
IENERGIA & Pequeno $(<5.000 \mathrm{GWh})$ & Sul \\
LIGHT & Grande $(>5.000 \mathrm{GWh})$ & Sudoeste \\
MUXENERGIA & Pequeno $(<5.000 \mathrm{GWh})$ & Sul \\
RGE & Grande $(>5.000 \mathrm{GWh})$ & Sul \\
UHENPAL & Pequeno $(<5.000 \mathrm{GWh})$ & Sul \\
\hline
\end{tabular}

